\title{
PREPRINT
}

P. Ripka, V. Grim, A. Chirtsov: A 3-Phase Current Transducer based on microfluxgate sensors, Measurement Vol. 146, November 2019, pp. 133-138 https://doi.org/10.1016/j.measurement.2019.06.028

\section{A 3-Phase Current Transducer based on microfluxgate sensors}

\section{Pavel Ripka*, Václav Grim and Andrey Chirtsov}

Czech Technical University in Prague, Faculty of Electrical Engineering, Technicka 16027 Praha 6, Czechia; ripka@fel.cvut.cz (P.R.); vaclav.grim@fel.cvut.cz (V.G.); chirtand@fel.cvut.cz (A.C.)

* Corresponding author: ripka@fel.cvut.cz

\begin{abstract}
Novel 3-phase DC/AC current transducer for 3-phase current lines is based on 8 integrated fluxgate sensors. Using full information from each sensor rather than gradiometric pairs, we suppress crosstalk between individual phases and external magnetic fields up to the 4 th order gradients. The suppression of external currents at a distance of $1 \mathrm{~m}$ is $90 \mathrm{~dB}$. The main advantage of the used microfluxgates is excellent temperature stability allowing operation outside the laboratory: the achieved temperature coefficient of the sensitivity is $50 \mathrm{ppm} / \mathrm{K}$, and the offset stability is $1 \mathrm{~mA} / \mathrm{K}$. These values are 20-times better than temperature stability achievable with current transducers sensors based on Hall sensors and magnetoresistors.
\end{abstract}

Keywords: current measurement; fluxgate; power distribution

\section{Introduction}

Fast and precise control of modern electric grids requires to measure voltage and current values at multiple points. [1]. This requires to deploy large number of new sensors on existing power lines and stations. The ability to measure the current DC component is required, as DC electric current may saturate current measurement transformers, leading to large measurement errors and possible failures. The DC current component is induced from geomagnetic variations and is also caused by transients, by transformerless inverters, and by induction from current changes in DC power lines [2].

In order to reduce the installation costs, ideal sensors are light so that they do not need their own supporting structure. The current sensor that is most widely used in electric grids is the current transformer. Some weight reduction can be achieved by using nanocrystalline alloys with high saturation magnetization and high permeability [3]. The cores can be cut to allow installation without breaking the measured conductor [4]. However, current transformers are still heavy, and the costs for installing them on the existing lines are high. Traditional current transformers also cannot measure the DC component, although efforts have been made to measure it using the fluxgate principle [5]. DC currents can be measured by magnetic sensors in the airgap of the ferromagnetic yoke (core) around the measured conductor [6]. This is also a heavy device with high installation costs.

Rogowski coils are lightweight and if made flexible they can be easily mounted on existing conductors, but they measure only AC or pulse currents since they are based on the induction law. [7]. The B-Dot sensors work on the same principle, using flat coils made of multilayer PCB [8]. These sensors have wide bandwidth, but they are suitable only for measuring large AC currents, because 
the low number of coil turns limits their sensitivity at low frequency. Fiber optic current sensors based on Faraday effect are temperature sensitive and they have large noise [9].

Yokeless current transducers using magnetic sensor arrays have best potential for this application. Gradiometric sensors suppress the influence of homogeneous external fields. They can be used if the unmeasured external currents are far enough away for their gradient to be negligible [10], [11] . Increasing the number of sensors inside the busbar can reduce the crosstalk from external currents [12].

Circular sensor arrays are based on Ampère's law. If the number of sensors is large, the sum of their output approximates the line integral and the reading is independent on external magnetic fields. To achieve this, 6 or 8 sensors are required for each phase [13] [14] [15-17]. Another advantage of these multisensor arrays is that they are immune to position changes of the measured conductor [18]. Sensors in the array may be divided into gradiometric pairs. Using three gradiometric pairs, error of $0.3 \%$ was achieved for external currents in the distance of $30 \mathrm{~cm}$ [19].

Hall sensors, Lorenz-force sensors, Anisotropic magnetoresistors (AMR) and Giant magnetoresistors (GMR), and also microfluxgate sensors, are magnetic sensors that are suitable for yokeless current transducers. Hall sensors are used in [10], and in [14]. Thanks to their range up to $2 \mathrm{~T}$, they can measure very high currents in the kA range. However, they suffer from poor DC stability and, in addition, their sensitivity is temperature-dependent. AMR sensors were used in [16, 20], and [21]. AMR sensors have a limited dynamic range: low-noise sensors can measure fields only up to 0.2 $\mathrm{mT}$ [22]. GMR sensors still suffer from limited linearity. TMR sensors were used in [19]. They may have $1 \%$ linearity and hysteresis in the linear range of $0.5 \mathrm{mT}$, and their frequency response is up to $\mathrm{MHz}$ range [23], but their weak point is poor DC stability. MEMS sensors based on Lorentz force sensors and also sensors based on the force effect on a permanent magnet [24] have high linearity and a very high field range, but they still have high noise [25]. High noise and poor DC stability are also attributes of magnetostriction sensors [26] and sensor based on shape memory alloy [27]. Microfluxgate sensors are used in [11,12], and [15]. They have $2 \mathrm{mT}$ range, a dynamic range similar to that of AMR sensors, but they have excellent temperature stability of both offset and sensitivity. Other sensor types such as magnetoelectric [28] or force-based [29] have been also used for current transducers, but without achieving competitive parameters.

The application of a yokeless current transducer for measurements in a 3-phase network was suggested only recently, in [21]. The authors use 6 Anisotropic Magnetoresistance (AMR) sensors in 3 gradiometric pairs, and each pair measures the phase current from a close distance of $30 \mathrm{~mm}$. Although this simple solution suppresses the external homogeneous field, it does not suppress the field gradients, which are not negligible for close external currents. The simple gradiometric configuration also suffers from crosstalk between the phases.

The first version of our improved 3-phase transducer, using 6 sensors, was described in [30] . Our application also requires the DC current component to be measured. We therefore decided to use microfluxgate sensors, which have better DC offset stability than AMR sensors. Instead of calculating 3 field gradients, we performed more complex processing of the sensor outputs. As a result, the suppression of external currents was improved 15-fold in comparison with [21].

In this paper, we present an improved version of our design, using 8 sensors. We will show both theoretically and experimentally that much better suppression can be achieved with the new hardware. However, individual sensor calibration is necessary, and also corrections for sensor mismatch.

The paper is organized as follows: In Section 2, we describe several geometrical configurations of the sensors measuring the currents in the 3-phase power line, and we define the calculation 
methods for evaluating the measured currents. In section 3, we describe a model of three-phase power lines and associated instrumentation used for testing. The calibration method is described in section 4. In Section 5, the theoretical results for crosstalk are compared with the measurements. Sensor noise and temperature stability are discussed in Section 6. 
2. Description of Measurement Methods

We assume a simple linear three-phase overhead power line with the distance between conductors $d=30 \mathrm{~cm}$. For the measurement we use an array of lightweight magnetic sensors mounted in plane perpendicular to the conductors.

\subsection{Asymmetrical gradient method}

This configuration was described in [21] and also used in [19] . It uses pairs of close sensors (Fig. 1a) to calculate the gradients (or more precisely the differences) $H_{2}-H_{1}, H_{4}-H_{3}$, and $H_{6}-H_{5}$, which are then used to calculate the phase currents values, while the external fields are partly suppressed.

If only $\mathrm{I}_{1}$ current is present, then $\mathrm{H}_{1}=\mathrm{I}_{1} / 2 \pi \mathrm{r}_{2}, \mathrm{H}_{2}=\mathrm{I}_{1} / 2 \pi \mathrm{r}_{1}$ and we can write

$$
I_{1 \text { true }}=2 \pi \frac{\left(H_{2}-H_{1}\right)}{\left(\frac{1}{r_{1}}-\frac{1}{r_{2}}\right)}
$$

If only $\mathrm{I}_{2}$ is present, then $\mathrm{H}_{1 \text { false }}=\mathrm{I}_{2} / 2 \pi\left(\mathrm{r}_{1}+\mathrm{d}\right)$ and $\mathrm{H}_{2 \mathrm{false}}=\mathrm{I}_{2} / 2 \pi\left(\mathrm{r}_{2}+\mathrm{d}\right)$.

Now we can evaluate the crosstalk as

$$
\frac{I_{\text {false }}}{I_{\text {true }}}=\frac{\Delta H_{\text {false }}}{\Delta H_{\text {true }}}=\frac{\frac{1}{d+r_{2}}-\frac{1}{d+r_{1}}}{\frac{1}{r_{2}}-\frac{1}{r_{1}}}
$$

where true values are caused by current $\mathrm{I}_{1}$, and false field and current readings are caused by current $\mathrm{I}_{2}$ of the same size.
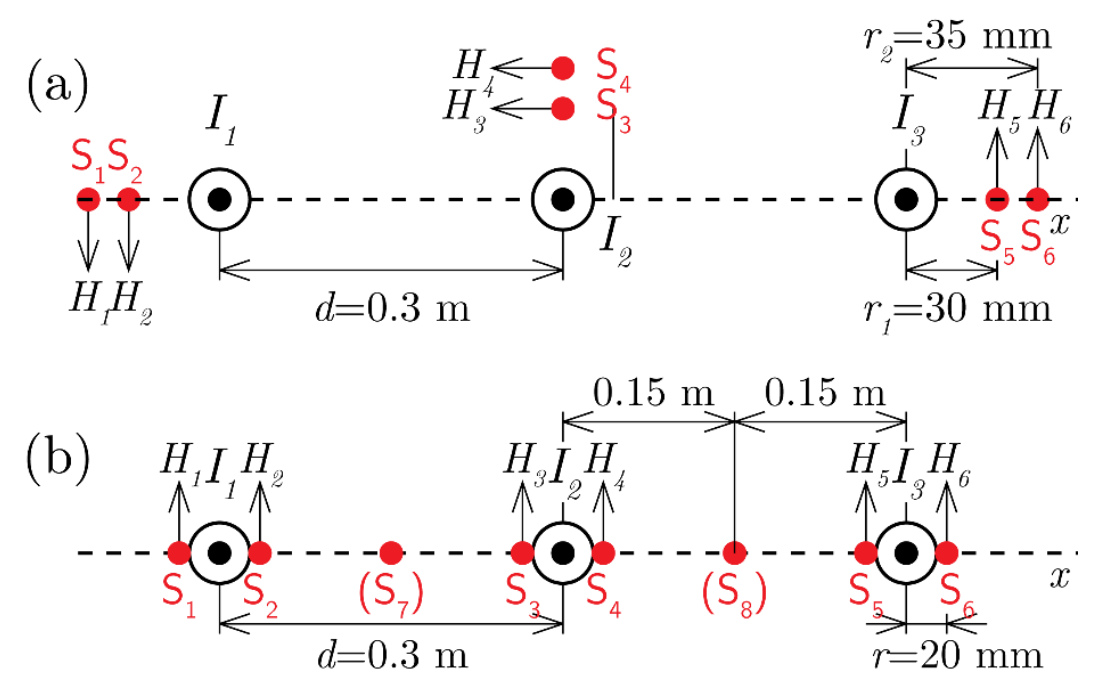

Figure 1. Placement of the sensors (a) for the asymmetrical gradient method [21], and (b) for all other methods (dimensions not to scale).

For the dimensions from Fig. 1a, the crosstalk ratio is $0.95 \%$ or $-40 \mathrm{~dB}$. The suppression of external currents can be calculated using (2), if we replace $d$ by the distance of the external current to the respective conductor. The results are plotted in Fig. 6. 
The disadvantage of this method lies in the loss in sensitivity caused by the fact that in (2) we subtract two similar values, $\mathrm{H}_{1}$ and $\mathrm{H}_{2}$.

\subsection{Symmetrical gradient method}

This configuration uses two sensors at each phase wire, located symmetrically at each side of the measured conductor at a distance $r=20 \mathrm{~mm}$ (as shown in Fig. 1b). Differential method again suppress an external homogeneous field component (such as the Earth's field).

If only $\mathrm{I}_{1}$ current is present, then

$\mathrm{H}_{1}=\mathrm{I}_{1} / 2 \pi \mathrm{r}, \mathrm{H}_{2}=-\mathrm{I}_{1} / 2 \pi \mathrm{r}, \mathrm{I}_{1 \text { true }}=\left(\mathrm{H}_{1}-\mathrm{H}_{2}\right) \pi \mathrm{r}$,

If only $\mathrm{I}_{2}$ is present, then $\mathrm{H}_{1 \text { false }}=\mathrm{I}_{2} / 2 \pi(\mathrm{d}+\mathrm{r})$ and $\mathrm{H}_{2 \text { false }}=\mathrm{I}_{2} / 2 \pi(\mathrm{d}-\mathrm{r})$.

and for the crosstalk ratio from the nearest phase we can write

$$
\frac{I_{\text {false }}}{I_{\text {true }}}=\frac{r}{d+r}-\frac{r}{d-r}
$$

For the dimensions from Fig. 1, the crosstalk ratio from the nearest phase is $0.9 \%$, i.e. similar to that for the asymmetrical gradient method. The advantage is that the sensitivity is higher than when the first method is used, resulting in smaller error and less noise. This is due to the fact that $\mathrm{H}_{1}$ and $\mathrm{H}_{2}$ have opposite signs. However, the larger sensor distance results in lower immunity to field gradients.

The suppression of external currents can be calculated using (3), if we replace $d$ by the distance of the external current from the respective conductor. The results are again plotted in Fig. 6 .

\subsection{Method with Polynomial Estimation}

The last method corrects the disadvantages of both previous configurations. We use the same arrangement as is presented in Fig. 1b. Instead of calculating the field gradients, we utilize the output of all sensors in a more complex way.

From the readings of six sensors we can calculate three unknown phase currents and the position and amplitude of one external current. If the currents are DC, we have 6 independent equations, and for AC current we have 6 equations for instantaneous values, or for a sinewave waveform we have 6 equations for amplitude and 6 for phase.

However, we cannot always assume that there will be only a single external current, and we should consider the effect of external fields of other origin, such as ferromagnetic objects, which can also distort the field from an external current. We therefore decided to find a universal solution that nulls the external field in the $y$-direction and its gradients up to the second order. Fields in the $x$ direction do not affect the sensors at all. We therefore suppose that the field at each sensor consists of three contributions from the phase currents and a contribution from the external field Hext

$$
H_{n}=\frac{I_{1}}{2 \pi\left(S_{n x}-W_{1 x}\right)}+\frac{I_{2}}{2 \pi\left(S_{n x}-W_{2 x}\right)}+\frac{I_{3}}{2 \pi\left(S_{n x}-W_{3 x}\right)}+H_{e x t}(x, y, t)
$$

where $H_{n}$ is field intensity at sensor $n, I_{1}-I_{3}$ are (unknown) phase currents, $S_{n x}$ is the x-coordinate of sensor $n$, and $W_{n x}$ is the $x$-coordinate of each phase conductor. The solution does not depend on the placement of the origin of the coordinate system; here $W_{3 x}=0$ was assumed. 
External field $H_{\text {ext }}$ may have any spatial distribution, which we approximate with a fourth degree polynomial:

$$
H_{\text {ext }}(x) \approx H_{E 0}+H_{E 1} x+H_{E 2} x^{2}+H_{E 3} x^{3}+H_{E 4} x^{4}
$$

Six sensors give suppression up to $2^{\text {nd }}$ order gradient [30]. In order to null response up to $4^{\text {th }}$ order gradient, the number sensors should be increased to 8 . Two additional sensors, $S_{7}$ and $S_{8}$, are placed halfway between the phase conductors (Fig. 1b).

Eight sensors produce eight different equations [30]. A linear system is formed:

$$
\left(\begin{array}{l}
H_{1} \\
H_{2} \\
H_{3} \\
H_{4} \\
H_{5} \\
H_{6} \\
H_{7} \\
H_{8}
\end{array}\right)=\left(\begin{array}{cccccccc}
\frac{1}{2 \pi\left(S_{1 x}-W_{1 x}\right)} & \frac{1}{2 \pi\left(S_{1 x}-W_{2 x}\right)} & \frac{1}{2 \pi\left(S_{1 x}-W_{3 x}\right)} & 1 & S_{1 x} & S_{1 x}^{2} & S_{1 x}^{3} & S_{1 x}^{4} \\
\frac{1}{2 \pi\left(S_{2 x}-W_{1 x}\right)} & \frac{1}{2 \pi\left(S_{2 x}-W_{2 x}\right)} & \frac{1}{2 \pi\left(S_{2 x}-W_{3 x}\right)} & 1 & S_{2 x} & S_{2 x}^{2} & S_{2 x}^{3} & S_{2 x}^{4} \\
\frac{1}{2 \pi\left(S_{3 x}-W_{1 x}\right)} & \frac{1}{2 \pi\left(S_{3 x}-W_{2 x}\right)} & \frac{1}{2 \pi\left(S_{3 x}-W_{3 x}\right)} & 1 & S_{3 x} & S_{3 x}^{2} & S_{3 x}^{3} & S_{3 x}^{4} \\
\frac{1}{2 \pi\left(S_{4 x}-W_{1 x}\right)} & \frac{1}{2 \pi\left(S_{4 x}-W_{2 x}\right)} & \frac{1}{2 \pi\left(S_{4 x}-W_{3 x}\right)} & 1 & S_{4 x} & S_{4 x}^{2} & S_{4 x}^{3} & S_{4 x}^{4} \\
\frac{1}{2 \pi\left(S_{5 x}-W_{1 x}\right)} & \frac{1}{2 \pi\left(S_{5 x}-W_{2 x}\right)} & \frac{1}{2 \pi\left(S_{5 x}-W_{3 x}\right)} & 1 & S_{5 x} & S_{5 x}^{2} & S_{5 x}^{3} & S_{5 x}^{4} \\
\frac{1}{2 \pi\left(S_{6 x}-W_{1 x}\right)} & \frac{1}{2 \pi\left(S_{6 x}-W_{2 x}\right)} & \frac{1}{2 \pi\left(S_{6 x}-W_{3 x}\right)} & 1 & S_{6 x} & S_{6 x}^{2} & S_{6 x}^{3} & S_{6 x}^{4} \\
\frac{1}{2 \pi\left(S_{7 x}-W_{1 x}\right)} & \frac{1}{2 \pi\left(S_{7 x}-W_{2 x}\right)} & \frac{1}{2 \pi\left(S_{7 x}-W_{3 x}\right)} & 1 & S_{7 x} & S_{7 x}^{2} & S_{7 x}^{3} & S_{7 x}^{4} \\
\frac{1}{2 \pi\left(S_{8 x}-W_{1 x}\right)} & \frac{1}{2 \pi\left(S_{8 x}-W_{2 x}\right)} & \frac{1}{2 \pi\left(S_{8 x}-W_{3 x}\right)} & 1 & S_{8 x} & S_{8 x}^{2} & S_{8 x}^{3} & S_{8 x}^{4}
\end{array}\right) \cdot\left(\begin{array}{c}
I_{1} \\
I_{2} \\
I_{3} \\
H_{E 0} \\
H_{E 1} \\
H_{E 2} \\
H_{E 3} \\
H_{E 4}
\end{array}\right)
$$

Solving this matrix equation (using the default Matlab solver) provides all currents and a 1-D estimate of the external field (homogeneous component and first four gradients) at the same time.

Ideally, the crosstalk from the other two phases is zero.

The suppression of the external currents depends on the distance of the current in a complex way, and cannot be expressed analytically. We have calculated the suppression numerically for each distance, and the results are plotted in Fig. 4, together with the values calculated for the two gradiometric methods. It is clear that when using only 6 sensors the improvement over the asymmetrical gradiometric method is only slight: by a factor of 15 for very short distances and by a factor of 30 for a distance of $1 \mathrm{~m}$. The reason for this is that the field from the external conductor is rich in higher-order gradients, which are not nulled. Using 8 sensors shows best theoretical suppression.

In order to test how our method is robust against uncertainty in the position of the sensors and their sensitivity, we have made sensitivity analysis using Monte Carlo method. Position or gain of each sensor was varied randomly around its nominal value. Perturbations followed a normal distribution with small amplitude ( $\sigma=0.01 \%$ of nominal value). Sensor readings at corresponding locations were calculated and from them the value of $I_{3}$ was deduced. No correlation among dimensions or gains was assumed, i.e. each variable subject to perturbation is modified by a newly generated random number. Simulation was repeated 100000 times. Sensitivity coefficients shown in the following table are ratios of normalized standard deviations.

Table 1 shows that the sensitivities calculated for $\mathrm{I}_{3}$ (which is the measured current nearest to external current $I_{4}$ ) are less than 1 . The sensitivity to the sensor noise is the same as to the sensor gain variation, because the measured noise spectrum is close to AWGN approximation. 
Table 1. Sensitivity analysis of polynomial estimation method, external conductor $\mathrm{I}_{4}$ at $100 \mathrm{~cm}$

\begin{tabular}{l|c} 
Input quantity $\xi$ & Sensitivity $\left(\left(\% \mathrm{I}_{3}\right) /(\% \xi)\right)$ \\
\hline Wire-to-wire distance & 0.05 \\
Wire-to-sensor distance & 0.77 \\
Sensor gain variations & 0.78
\end{tabular}

\section{Measurement Setup}

The measurements were performed with 5 A rms phase currents supplied from a $50 \mathrm{~Hz}$ threephase transformer. The $4.4 \mathrm{~m}$ long straight power line was terminated by resistors. Sensor boards were mounted on wooden support (Fig. 2). TI DRV425 integrated microfluxgate sensors were used as vectorial field sensors (Fig. 3). 5 A external current was supplied from another transformer. The true current values were measured using $0.05 \%$ precise shunt resistors. The voltage drops across the shunts as well as sensor outputs were measured by NI 6211 16-bit A/D converter USB module with 5000 samples/s rate. All collected data was processed offline in Matlab and averaged over 10 periods.

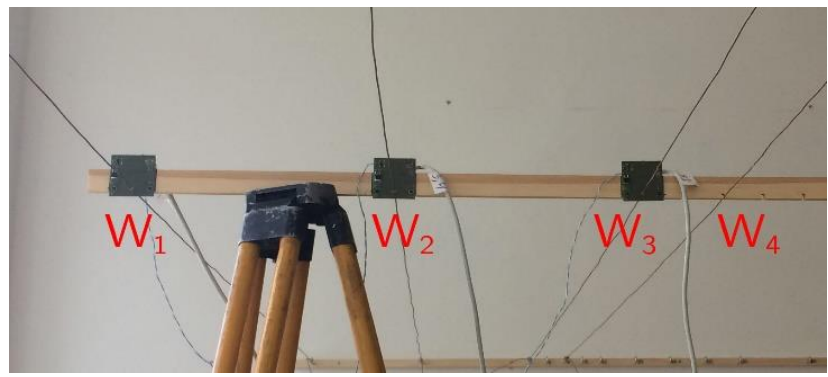

Figure 2. A photo of the measurement setup with $W_{4}$ at a distance of $10 \mathrm{~cm}$ from $W_{3 .}$ [30]

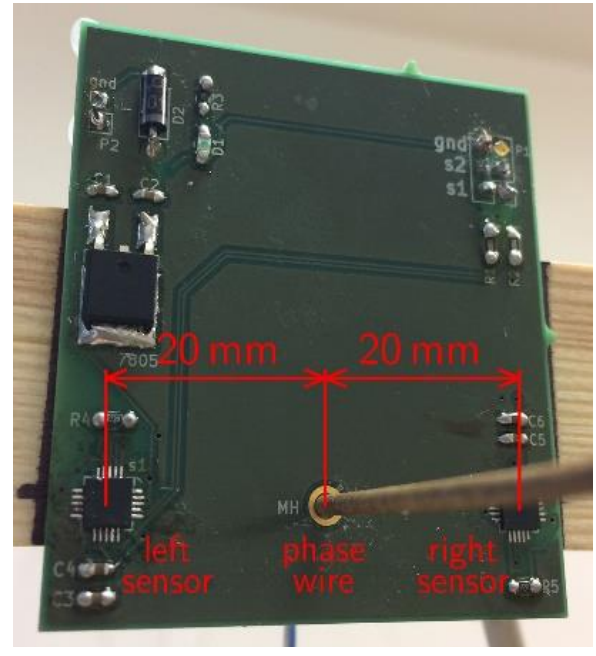

Figure 3. Detail of sensor PCB with a pair of DRV425s. [30]

\section{Calibration}

The sensitivity of each sensor was calibrated in Helmholtz coils using a simple system controlled by LabVIEW. DC current with alternating polarity was used to eliminate the effects of the 
geomagnetic field. Using this procedure, we are able to separate the influence of sensitivity errors and geometrical errors. Table 1 shows the sensitivity correction coefficients that were obtained.

Sensor calibration was also performed by passing a 5 A current successively through individual phase conductors. The results were very similar, indicating that the sensitivity mismatch is the dominating factor, and the geometrical errors are negligible. 
Table 1 Results of the sensitivity calibration.

\begin{tabular}{ccc}
\hline sensor \# & correction factor & sensitivity deviation (\%) \\
\hline 1 & 0.974748 & -2.52 \\
2 & 0.974569 & -2.54 \\
3 & 0.97903 & -2.09 \\
4 & 0.97062 & -2.94 \\
5 & 1.0605 & 6.05 \\
6 & 1.03849 & 3.85 \\
7 & 0.98495 & -1.51 \\
8 & 0.988595 & -1.14 \\
\hline
\end{tabular}

\section{Calculated and Measured Results}

A comparison of the calculated results shows that our new polynomial estimation method has an advantage over both gradient methods (described in Sections 2.1 and 2.2.). Fig. 4 summarizes the calculated theoretical rejection of the external current in the x-direction. At greater distances, increasing the number of sensors from 6 to 8 significantly increases the rejection. For the external current at a distance of $1 \mathrm{~m}$ and using a polynomial method with 8 sensors, the crosstalk from the external currents is theoretically 0.001 , compared with 0.1 which can be achieved by the asymmetrical gradient configuration suggested in [21].

We also analyzed the influence of the angular position of the external current: we observe that the maximum error is for external currents in the $\mathrm{x}$ - and y-directions. This is clear from Fig. 5, which shows the rejection of the external current at a distance of $50 \mathrm{~cm}$, as a function of its angular position. Zero error is reached for the external current in the $45^{\circ}$ direction.

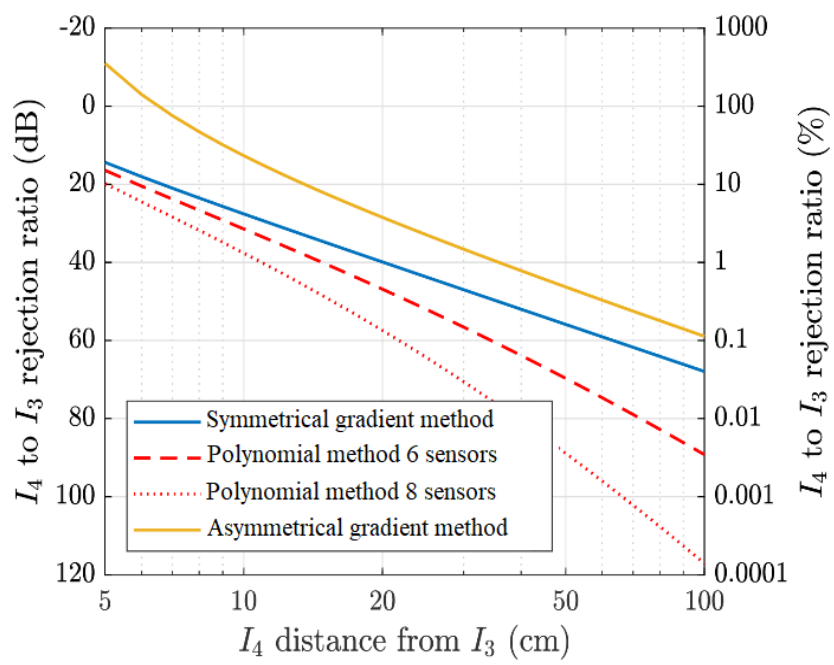

Figure 4. Suppression of external current in the $x$ direction as a function of its distance: A theoretical comparison of various current measurement methods. 


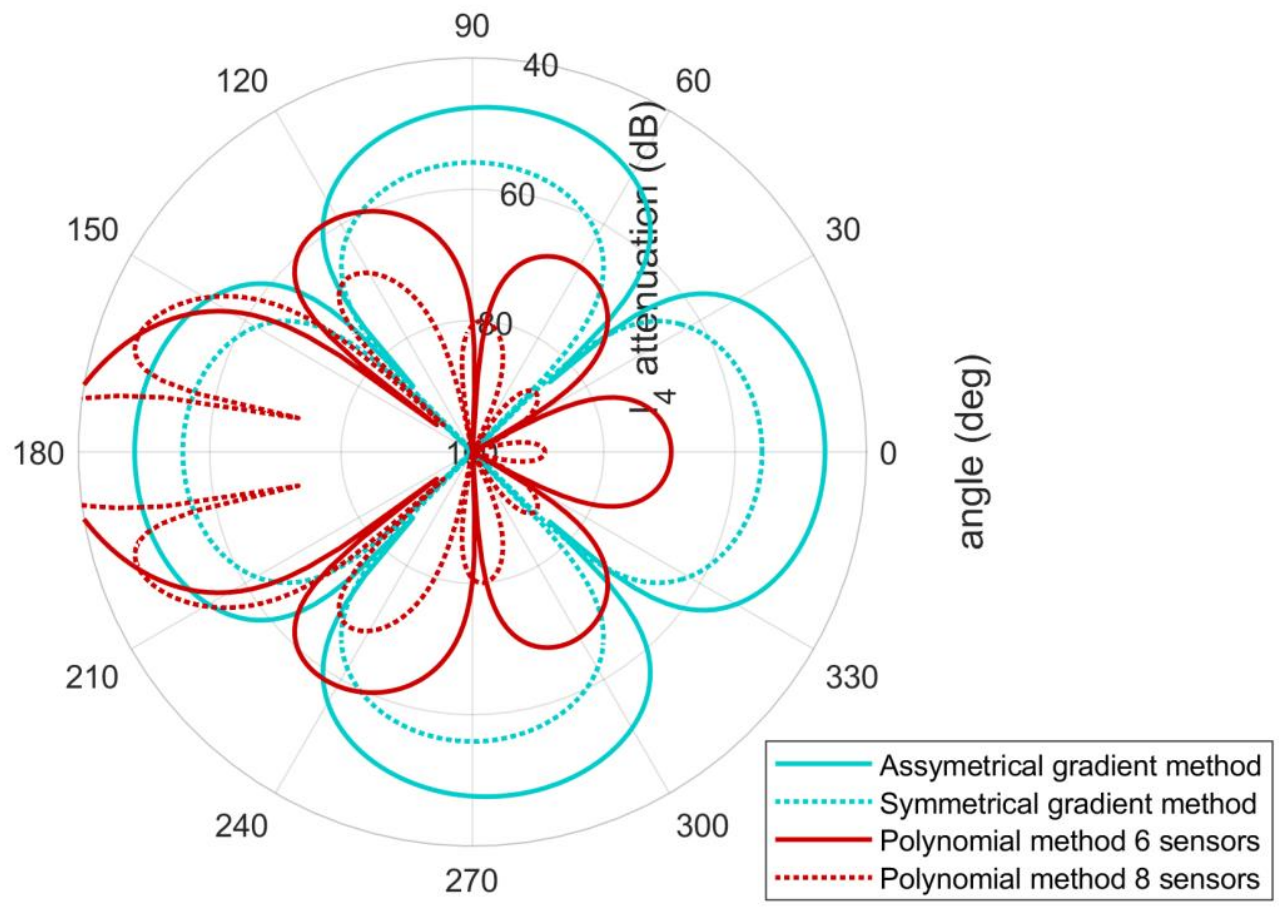

Figure 5. Suppression of external current at a distance of $50 \mathrm{~cm}$ as a function of the angular position (calculated values).

Fig. 6 shows the measured rejection ratio of the external current as a function of distance. While for 6 sensors the measured values correspond well with the simulations in Fig. 6, the rejection ratio of the 8-sensor transducer is smaller than expected. After a detailed analysis, we found that an 8sensor transducer is much more sensitive to the mismatch of sensor parameters. We calibrated individual sensors, and after a correction had been made for their sensitivities, the rejection ratio improved significantly, as shown in Fig. 7. The measured rejection at a distance of $1 \mathrm{~m}$ was $80 \mathrm{~dB}$ (crosstalk $0.003 \%$ ), i.e. a 300-fold improvement in comparison with [21].

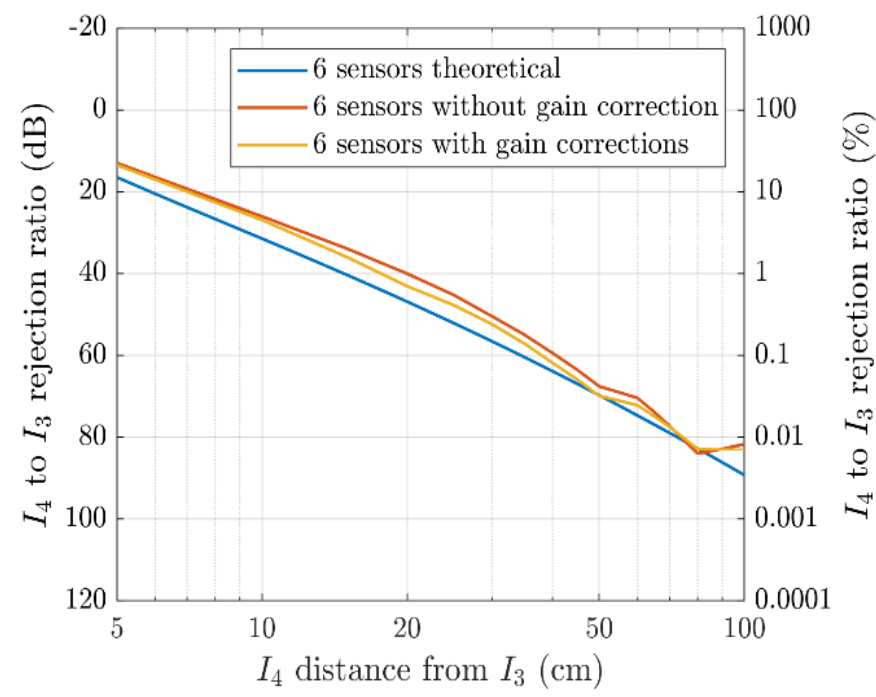

Figure 6. Measured suppression of external current in the $x$ direction as a function of its distance for setup with 6 sensors, with and without gain corrections. 


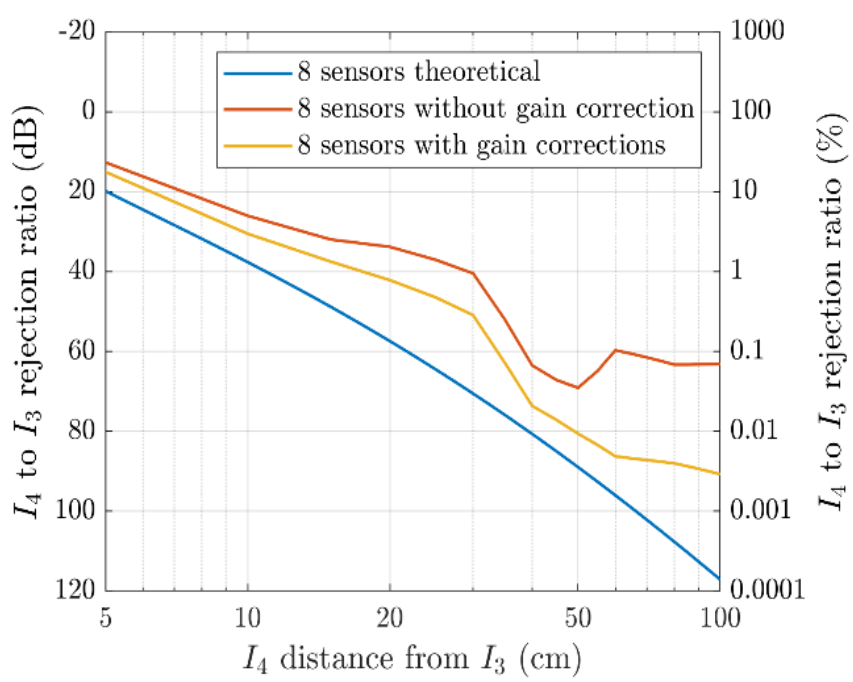

Figure 7. Measured suppression of external current in the $x$ direction as a function of its distance for setup with 8 sensors, with and without corrections.

\section{Noise, Temperature Stability and Linearity}

The application of our sensors is the measurement of geomagnetically induced currents in long power lines. These currents are DC with rate of change in minutes. Using traditional DC current sensors in outdoor environment resulted in unacceptable temperature drifts: with AMR sensors the transducer offset drift was $50 \mathrm{~mA} / \mathrm{K}$ and the sensitivity temperature coefficient was $0.3 \% / \mathrm{K}$. By replacing AMR by microfluxgate sensor, these values were improved to $1 \mathrm{~mA} / \mathrm{K}$ and $50 \mathrm{ppm} / \mathrm{K}$. These values were calculated from the datasheet of the manufacturer and verified by measurement at 3 temperatures.

Sensor noise was measured with the use of two different methods: the noise of the individual sensor was measured using SR770 spectrum analyzer. The achieved noise power density was 2.5 $\mathrm{nT} / \sqrt{ } \mathrm{Hz}$ at $1 \mathrm{~Hz}$, corresponding to ideal noise level of $0.25 \mathrm{~mA} / \sqrt{ } \mathrm{Hz}$ at $1 \mathrm{~Hz}$. The noise of the complete transducer was measured using Matlab FFT calculation from sampled data (Fig. 8). Measurements were performed at room temperature $\left(20\right.$ to $\left.25^{\circ} \mathrm{C}\right)$. In both cases, a three-layer permalloy shield was used. The noise power spectrum density of the complete transducer including the A/D converter is $0.4 \mathrm{~mA} / \sqrt{ } \mathrm{Hz}$ at $1 \mathrm{~Hz}$. For frequencies higher than $1 \mathrm{~Hz}$ the noise spectrum is white - the RMS noise level from $1 \mathrm{~Hz}$ to $1 \mathrm{kHz}$ is $6 \mathrm{~mA}$. This is comparable to the noise level of transducer based on precise AMR sensors. 


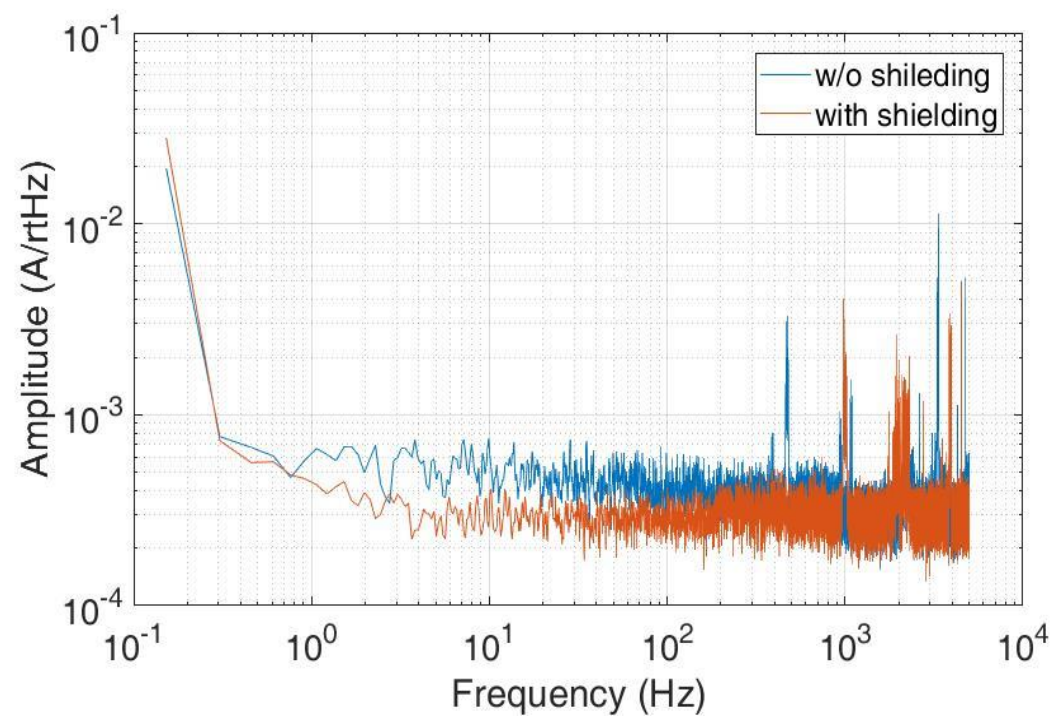

Figure 8. Measured noise spectrum of the complete transducer inside the shielding and without shielding.

Another advantage of the proposed sensor is high linearity which is limited by $0.1 \%$ linearity error of individual feedback compensated microfabricated fluxgate sensors. The measured linearity error of the complete transducer is shown in Fig. 9.

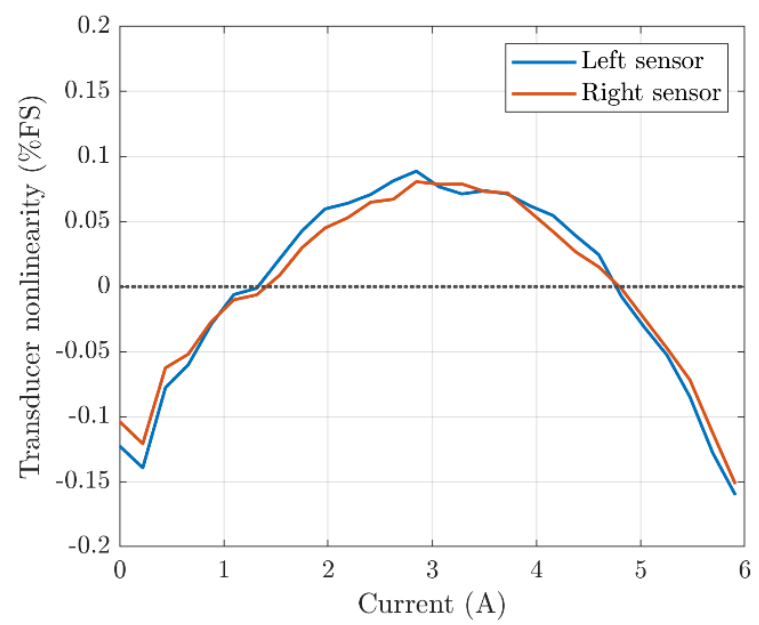

Figure 9. Linearity of the complete transducer

\section{Discussion}

We described optimized microfluxgate current transducer suitable for monitoring geomagnetically induced currents and other DC current components in 3-phase current lines The traditional way to process the sensor data, which we call the "gradient method", calculated the differences of two sensors in a gradiometric pair. However, this method lost part of the information. 
The gradiometric method suppressed only the homogeneous component of the external field, not the field gradients. For external (non-measured) currents within a small distance the gradients are high, which results in poor suppression of these currents.

In our novel method, we utilize the output of all sensors in a more complex way by solving equation (4). First of all, we completely compensate the crosstalk between the phases. Using six sensors, we also suppress the first and second gradient of the external field.

By using 8 sensors we can suppress field gradients up to the 4 th order. This results in even larger suppression of external currents. Sensor calibration is necessary in order to achieve the projected parameters for an 8-sensor transducer. The measured rejection at $1 \mathrm{~m}$ distance was $90 \mathrm{~dB}$ (or $0.003 \%)$, i.e. a 300-fold improvement in comparison with [21]. By Monte Carlo simulations we verified that the method is robust against uncertainty of sensor position and sensor data.

Replacing magnetoresistive sensors by integrated fluxgate sensors improved the temperature stability 50-fold: $1 \mathrm{~mA} / \mathrm{K}$ offset stability and $50 \mathrm{ppm} / \mathrm{K}$ temperature coefficient of sensitivity of the developed current transducer was achieved. The transducer noise is $6 \mathrm{~mA}$ RMS (1Hz to $1 \mathrm{kHz})$ and linearity error is $0.15 \%$ in 6 A range.

LEM LF210, which has $20 \mathrm{~mA}$ RMS noise, 0,15 A offset and $75 \mathrm{~g}$ mass for single phase, is an example of an available DC/AC current transducer with the same 200 A range. Compared to that, our sensor is lightweight (12 g for each phase). It can therefore be mounted on existing overhead 3-phase current lines with low installation costs. It also has excellent temperature stability and low noise.

\section{Acknowledgements}

This work was supported by the Grant Agency of the Czech Republic within the New Methods for the Measurement of Electric Currents" project (GACR 17-19877S).

\section{References}

[1] Salman, and K. Salman, Introduction to the Smart Grid: The Institution of Engineering and Technology, 2017.

[2] A. Pulkkinen, E. Bernabeu, A. Thomson et al., "Geomagnetically induced currents: Science, engineering, and applications readiness," Space Weather-the International Journal of Research and Applications, vol. 15, no. 7, pp. 828-856, Jul, 2017.

[3] W. Pluta, C. Swieboda, J. Leszczynski et al., "Some remarks on metrological properties and production technology of current transformers made of nanocrystalline cores," Measurement, vol. 97, pp. 38 - 44, 2017.

[4] C. Swieboda, J. Walak, M. Soinski et al., "Nanocrystalline oval cut cores for current instrument transformer prototypes," Measurement, vol. 136, pp. 50 - 58, 2019.

[5] P. Ripka, K. Draxler, and R. Styblíková, "DC-Compensated Current Transformer," Sensors, vol. 16, 2016, 2016.

[6] X. Cheng, Z. Sun, X. Wang et al., "Open-loop linear differential current sensor based on dualmode Hall effect," Measurement, vol. 50, pp. 29 - 33, 2014.

[7] A. Cataliotti, V. Cosentino, D. Di Cara et al., "Rogowski coil current transducer compensation method for harmonic active power error," Measurement, vol. 63, pp. 240-251, Mar, 2015.

[8] J. G. Wang, D. C. Si, T. Tian et al., "Design and Experimental Study of a Current Transformer with a Stacked PCB Based on B-Dot," Sensors, vol. 17, no. 4, Apr, 2017.

[9] Z. P. Wang, and X. Z. Wang, "Theoretical analysis of the temperature characteristics of an optical current sensing element," Measurement, vol. 42, no. 2, pp. 277 - 280, 2009. 
[10] M. Blagojevic, U. Jovanovic, I. Jovanovic et al., "Realization and optimization of bus bar current transducers based on Hall effect sensors," Measurement Science and Technology, vol. 27, no. 6, Jun, 2016.

[11] P. Ripka, and A. Chirtsov, "Influence of External Current on Yokeless Electric Current Transducers," Ieee Transactions on Magnetics, vol. 53, no. 11, pp. 4, Nov, 2017.

[12] P. Ripka, and A. Chirtsov, "Busbar Current Transducer With Suppression of External Fields and Gradients," Ieee Transactions on Magnetics, vol. 54, no. 11, Nov, 2018.

[13] L. Di Rienzo, and Z. Zhang, "Spatial Harmonic Expansion for Use With Magnetic Sensor Arrays," Ieee Transactions on Magnetics, vol. 46, no. 1, pp. 53-58, Jan, 2010.

[14] Y. P. Tsai, K. L. Chen, Y. R. Chen et al., "Multifunctional Coreless Hall-Effect Current Transformer for the Protection and Measurement of Power Systems," Ieee Transactions on Instrumentation and Measurement, vol. 63, no. 3, pp. 557-565, Mar, 2014.

[15] R. Weiss, R. Makuch, A. Itzke et al., "Crosstalk in Circular Arrays of Magnetic Sensors for Current Measurement," Ieee Transactions on Industrial Electronics, vol. 64, no. 6, pp. 49034909, Jun, 2017.

[16] P. Mlejnek, and P. Ripka, "AMR yokeless current sensor with improved accuracy," Proceedings of the 30th Anniversary Eurosensors Conference - Eurosensors 2016, Procedia Engineering I. Barsony, Z. Zolnai and G. Battistig, eds., pp. 900-903, 2016.

[17] P. Mlejnek, and P. Ripka, "Off-Center Error Correction of AMR Yokeless Current Transducer," Journal of Sensors, 2017, 2017.

[18] H. Yu, Z. Qian, H. Y. Liu et al., "Circular Array of Magnetic Sensors for Current Measurement: Analysis for Error Caused by Position of Conductor," Sensors, vol. 18, no. 2, pp. 12, Feb, 2018.

[19] Y. Chen, Q. Huang, A. H. Khawaja et al., "A novel non-invasion magnetic sensor array based measurement method of large current," Measurement, vol. 139, pp. 78 - 84, 2019.

[20] P. Mlejnek, and P. Ripka, "Off-Center Error Correction of AMR Yokeless Current Transducer," Journal of Sensors, pp. 7, 2017.

[21] A. Bernieri, L. Ferrigno, M. Laracca et al., "An AMR-Based Three-Phase Current Sensor for Smart Grid Applications,” Ieee Sensors Journal, vol. 17, no. 23, pp. 7704-7712, Dec, 2017.

[22] L. Jogschies, D. Klaas, R. Kruppe et al., "Recent Developments of Magnetoresistive Sensors for Industrial Applications," Sensors, vol. 15, no. 11, pp. 28665-28689, Nov, 2015.

[23] M. Dabek, P. Wisniowski, P. Kalabinski et al., "Tunneling magnetoresistance sensors for high fidelity current waveforms monitoring," Sensors and Actuators a-Physical, vol. 251, pp. 142147, Nov, 2016.

[24] Kahr, M., M. Stifter et al., "Dual Resonator MEMS Magnetic Field Gradiometer," Sensors, vol. 19, 2019.

[25] W. He, Y. R. Lu, C. W. Qu et al., "A non-invasive electric current sensor employing a modified shear-mode cymbal transducer," Sensors and Actuators a-Physical, vol. 241, pp. 120-123, Apr, 2016.

[26] G. F. Lou, X. J. Yu, and R. Ban, "A wide-range DC current sensing method based on disk-type magnetoelectric laminate composite and magnetic concentrator," Sensors and Actuators aPhysical, vol. 280, pp. 535-542, Sep, 2018.

[27] S. Pisupati, D. Kundukoori, N. Mekala et al., "Design of resonance based DC current sensor using BAW quartz resonators," Sensors and Actuators a-Physical, vol. 271, pp. 104-110, Mar, 2018.

[28] D. L. Shi, Q. Zhang, Y. M. Ye et al., "High-frequency current sensor based on lead-free multiferroic BiFeO3-BaTiO3-based ceramics,” Measurement, vol. 104, pp. 287-293, Jul, 2017.

[29] B. Santhosh Kumar, K. Suresh, U. Varun Kumar et al., "Resonance based DC current sensor," Measurement, vol. 45, no. 3, pp. 369 - 374, 2012.

[30] P. Ripka, G. V, and C. A, "Improved 3-Phase Current Transducer," Proceedings MDPI 2018, 2(13), 1070. 
